\title{
Evaluación de los Efectos de Diversas Intensidades de Estimulación Nerviosa Sobre la Amplitud de la Respuesta en Diferentes Especies
}

\author{
Evaluation of the Effects of Various Intensities of Nerve Stimulation \\ on the Scale of the Response in Different Species
}

Luis Miguel Ramírez Aristeguieta

\begin{abstract}
RAMÍREZ, L M. Evaluación de los efectos de diversas intensidades de estimulación nerviosa sobre la amplitud de la respuesta en diferentes especies. Int. J. Morphol., 28(1):227-238, 2010.

RESUMEN: El comportamiento nervioso a la estimulación eléctrica ha sido ampliamente estudiado desde 1840, época en la que se comienza a entender las características de la amplitud de respuesta nerviosa y velocidades de conducción en nervios de animales. La amplitud de respuesta en segmentos de nervios es producto de los potenciales de acción compuestos resultantes a la estimulación eléctrica. En esta investigación se estudió el comportamiento nervioso a potenciales evocados in vivo en humanos (nervio ulnar) e in vitro como nervio aislado en Bufus marinus. Durante la estimulación de los nervios se encontró respuesta de comportamiento cualitativo creciente y heterogéneo intra e ínter-especie. El análisis de las respuestas y mecanismos que participan en estos resultados se discuten.
\end{abstract}

PALABRAS CLAVE: Potencial de acción compuesto, Electrodo de estimulación, Electrodo de registro, trenes de estimulación, Intensidad de carga desplazada.

\section{INTRODUCCIÓN}

Las fibras de diámetros diferentes que forman un nervio generan potenciales de acción individuales que se suman para dar un potencial de acción compuesto que corresponde a la amplitud de respuesta del total de fibras de un nervio con diferentes velocidades de conducción (Nakanishi et al.,). Con el osciloscopio de rayos catódicos, Gasser y Erlanger (1922) pudieron registrar varios picos en el potencial de acción registrado del nervio ciático de rana (Frank, 1986).

La caracterización de las respuestas a la estimulación nerviosa para un nervio aislado compuesto de una morfología neuronal heterogénea debe entenderse ya no como subumbral, umbral y supraumbral para una sola neurona (siendo el umbral nervioso la intensidad de estimulo necesario para provocar la activación de una neurona expresada como potencial de acción), sino más bien como estimulorespuesta submaxima, máxima y supramaxima para un nervio compuesto por muchas neuronas (Bean, 2007; Caliandro et al., 2007). Se entiende que un estimulo submaximo es subumbral para algunas fibras no activadas por el potencial eléctrico evocado y umbral y/o supraumbral para las fibras del nervio activadas. Por lo tanto, estas respuestas registradas como potenciales de acción compuestos son técnicamente, potenciales de campo cuyas características pueden ser diversas dependiendo de algunos aspectos como los que serán descritos a continuación (Guyton, 2006).

Las evaluaciones de excitabilidad y conductividad de potenciales de acción compuestos pueden realizarse directamente sobre un nervio (aislado o in situ) o mediante inferencias como en el caso de la mayoría de las evaluaciones de neuroconducción motora realizada en humanos con fines clínicos. En este último caso, se suele aplicar una estimulación neuronal transdérmica y se registra la activación eléctrica del músculo inervado por ese nervio. Las inferencias sobre las características de la funcionalidad neuronal son obtenidas de las características de las respuestas musculares. El estudio del potencial de acción compuesto en nervio aislado, ofrece como ventaja el que las respues- 
tas eléctricas son obtenidas directamente del nervio activado, por tal motivo, esta observación directa permite análisis más específicos relacionados con la función neuronal.

La amplitud de las respuestas electrofisiológicas de órganos completos, en general, dependen de a) El número de fibras que componen el órgano b) La sincronización de la respuesta o si se quiere ser más preciso, la derivada de la respuesta (en voltios) en función del tiempo (dV/dt), lo que a su vez está fuertemente asociado a los umbrales y a las velocidades de conducción de las fibras individuales, c) La resistencia eléctrica entre el órgano activado y los electrodos de registro, lo que está muy relacionado (entre otras cosas) con la distancia y las propiedades de conductividad eléctrica de los tejidos interpuestos entre los electrodos de registro y el órgano activado, d) Del componente vectorial registrado en el potencial de campo, donde el paralelismo entre los dispositivos de registro bipolar y el vector de despolarización de un órgano determinan mayores amplitudes en las ondas de registro, y e) de las propiedades de los instrumentos de registro (Fukuda et al., 2007; Schuhfried, 2005).

De esta forma, si se evalúa de manera consecutiva las respuestas eléctricas musculares o nerviosas en un montaje experimental donde no cambien de ubicación los electrodos de estimulación y los de registro ni las propiedades del equipo de registro, las variaciones en las amplitudes de las respuestas dependerán esencialmente del número de fibras activadas en el órgano blanco y de la sincronización de las mismas.

Usualmente, la evaluación de la amplitud de la respuesta de un órgano excitable, está relacionada con el pico máximo del voltaje obtenido en la respuesta, sin embargo, tomando en consideración lo anteriormente expuesto, este valor estará íntimamente determinado por la máxima sincronización de las fibras de ese órgano, esto es, tal pico de voltaje ocurrirá por la sumatoria de los potenciales de campo obtenidos en el instante en que ocurrió ese pico. Esto puede subvalorar las activaciones de otras fibras del órgano que también han respondido frente al estímulo pero cuya respuesta no coincide temporalmente con las registradas en el momento del pico (Morélot-Panzini, 2009). En esa dirección, si se pretende obtener una idea más global de la activación de un órgano frente a estímulos conocidos, es probable que sea más conveniente, considerar el área bajo la curva de la onda de respuesta, es decir, la carga movilizada durante toda la respuesta. Igualmente podría ser útil tener parámetros de evaluación basados en la sincronización instantánea como los valores de las máximas derivadas (positivas y negativas) del voltaje en función del tiempo (Guyton).
La respuesta a un estimulo eléctrico se observa gráficamente como ondas que permiten evidenciar una despolarización en la que se disminuye la diferencia o gradiente de cargas del exterior y el interior celular seguida de una repolarizacion en la que se invierte este proceso y se recuperan los gradientes electroquímicos de las membranas nerviosas de cada neurona que compone este nervio y se logra alcanzar igualmente su potencial de reposo. En tal sentido la excitabilidad de un nervio y sus neuronas (o de cualquier órgano con células excitables) dependerá de la proximidad entre el potencial de reposo y los correspondientes umbrales de cada fibra individual lo que a su vez se traduce en un órgano completo como el nervio o el músculo, en potenciales de campo submáximos, máximos o supramáximos de acuerdo a la cantidad de fibras reclutadas en la activación.

Tomando en consideración los elementos antes enunciados, el presente trabajo busco describir entre varias especies las variaciones de la amplitud (picos máximos y áreas bajo la curva) de las respuestas neuronales y musculares frente a diversas intensidades de estimulación nerviosa y al tiempo describir parámetros de sincronización instantánea en los protocolos estudiados.

\section{MATERIAL Y MÉTODO}

La electroestimulación y evaluación de la respuesta eléctrica se realizó mediante el equipo PowerLab 16/30, el cual permitió visualizar y cuantificar tanto los parámetros de la estimulación nerviosa como los parámetros en tiempo y voltaje de la respuesta nerviosa. El equipo además permitió el almacenamiento de los registros. Para la estimulación y registro de nervio ciático aislado el procedimiento requirió electrodos de estimulación y registro que se conectaron al equipo en los canales 0 y 1 respectivamente. Los terminales de estimulación y registro se acoplaron a una "cámara para nervio aislado" con solución Ringer-Sapo por debajo del nivel de los electrodos. Para los potenciales evocados EMG del nervio ulnar en humanos, se utilizó adicionalmente una unidad de aislamiento conectada al amplificador principal.

Procedimiento en humanos: Se realizó electroestimulación y evaluación de la respuesta electromiografica a la estimulación progresiva creciente del nervio Ulnar durante su trayecto posterior del epicondilo medial del humero. Los electrodos de registro EMG se ubicaron sobre la eminencia hipotenar en donde la mayoría de los miotomas del nervio ulnar se representan. Se evaluaron seis valores de intensidades de estímulos aplicados $(10,12,14,16,18$ y 20 
$\mathrm{mA}$ ) de forma aislada. Cada estímulo tuvo una duración de $2 \mathrm{~ms}$. Cada intensidad fue repetida 3 veces con el fin de evaluar reproducibilidad de los resultados dentro de cada preparación experimental. Los protocolos fueron desarrollados a la temperatura ambiente del laboratorio y los resultados no fueron corregidos segun la temperatura de cada participante.

Procedimiento en Bufo marinus: Cada nervio fue cuidadosamente disecado y montado en una cámara de nervio aislado bajo ambiente humedecido con una solución RingerAnfibio. Se evaluaron 35 valores de intensidades de estímulos aplicados de forma aislada (de 80 a $410 \mathrm{mV}$ ) y creciente. Cada estímulo tuvo una duración de $2 \mathrm{~ms}$. Cada intensidad fue repetida 3 veces con el fin de evaluar reproducibilidad de los resultados dentro de cada preparación experimental. El control negativo para este procedimiento se obtuvo al impregnar con epinefrina el nervio, obteniéndose el bloqueo de este y el silencio en los electrodos de registro.

Tomando en consideración la variabilidad individual de cada muestra examinada, se procedió a normalizar los valores de cada variable y cada individuo por el máximo valor de su propia respuesta. Todos los resultados presentados a continuación, están expresados como porcentaje de la respuesta máxima. Se utilizó el promedio de las 3 medidas de cada intensidad para la representación final de los resultados de cada nervio. Se hizo un análisis de correlación y se encontraron los mejores ajustes a funciones logarítmicas y/ o lineales entre las variables examinadas.

\section{RESULTADOS}

La electroestimulación se realizó en cuatro nervios ulnares de humano y cinco nervios aislados de Bufo marinus. La Fig. 1 muestra la amplitud de respuesta representado como área bajo la curva para los nervios aislados ciáticos de Bufo marinus y humanos.

Se observaron respuestas crecientes de amplitud bajo estímulos submáximos y una estabilización de las respuestas (saturación) bajo estímulos máximos. Se observó una relación lineal entre área bajo la curva (carga movilizada) y la amplitud máxima. Cada una de las respuestas a estímulos crecientes presentó una dispersión importante en todos sus valores de amplitud de respuesta. La grafica de máximas amplitudes de respuesta y área bajo la curva de la respuesta (Figura 1) para ciático y ulnar se presentan similares y correspondientes a respuestas de nervios morfológicamente similares (nervios mixtos).
Las velocidades máximas de despolarización $(\mathrm{dV}+/$ dt) y repolarización $(\mathrm{dV}-/ \mathrm{dt})$ muestran el comportamiento del nervio en la máxima pendiente de cada fase de potencial de acción compuesto frente al estimulo escalonado y creciente aplicado (Fig. 2).

Las velocidades máximas de repolarización del nervio aislado tienen la tendencia a ser mayores y ajustan significativamente a una tendencia logarítmica (sapos R2=0.9832, humanos R2=0.0.992) (Fig. 3).

Las correlaciones a dos colas $(\mathrm{p}=0.01)$ entre las variables evaluadas del nervio ciático y ulnar (Tabla I) mostraron valores significativos lo que coincide con las funciones logarítmicas y lineales calculadas en la Figura 3. Las respuestas de amplitud neuronal a estímulos escalonados aunque heterogéneo intra e ínter-especie se expresa de manera creciente y predecible en sentido cualitativo.

La representación grafica de las respuestas a los estímulos nerviosos llega a saturarse lo que significa que a partir de un punto de estimulación no existirán más respuestas crecientes (Fig. 4). Cuando se relacionan la máxima amplitud de respuesta y el área bajo la curva de la misma se puede observas como las máximas amplitudes de respuesta tienden igualmente a "saturarse" mientras que el área bajo la curva aún puede continuar incrementando

La amplitud de respuesta en iguales nervios del mismo espécimen (ipsi y contralateral) mostraron respuestas de amplitud diferentes. La Fig.a 5 muestra las diferencias en la respuesta de amplitud a la estimulación de nervios ciáticos derecho e izquierdo del mismo espécimen.

\section{DISCUSIÓN}

Durante la estimulación de los nervios se encontró respuesta de comportamiento cualitativo creciente y heterogéneo intra e ínter-especie. Los mecanismos que participan en las variaciones de la amplitud (picos máximos y áreas bajo la curva) de las respuestas neuronales y musculares frente a diversas intensidades de estimulación nerviosa podrían centrarse en fenómenos de membrana en las fases de despolarización y repolarización para cada nervio estudiado. Tomando en consideración que las respuestas de amplitud nerviosa pueden variar dependiendo del tamaño del nervio, de su contenido de mielina y de la temperatura los resultados fueron bastante homogéneos entre los grupos estudiados.

Las respuestas nerviosas bajo los estímulos submaximos correspondieron a la fase de reclutamiento de 


\section{Máxima amplitud de la respuesta}

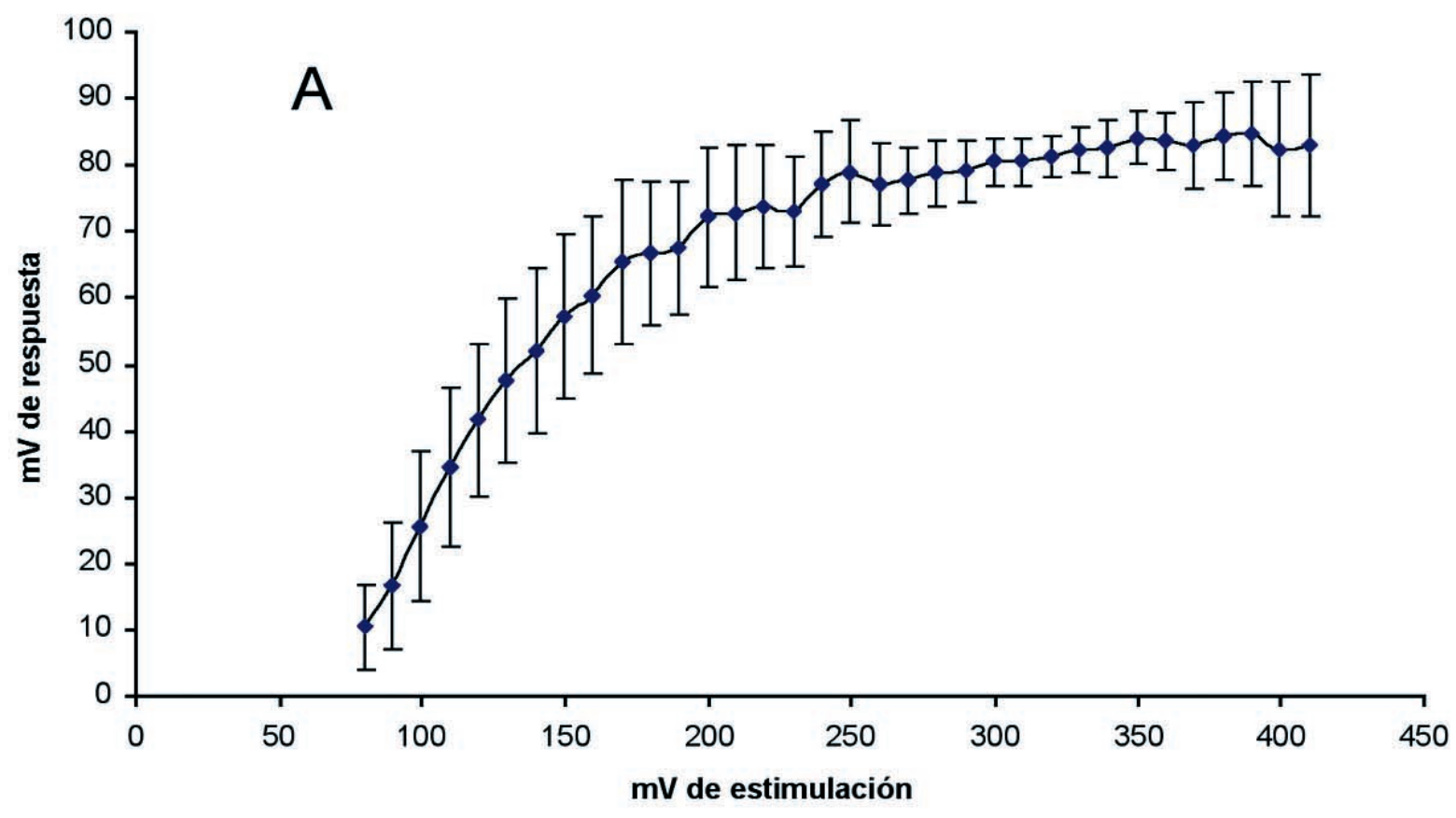

Area bajo la curva de la respuesta neuronal

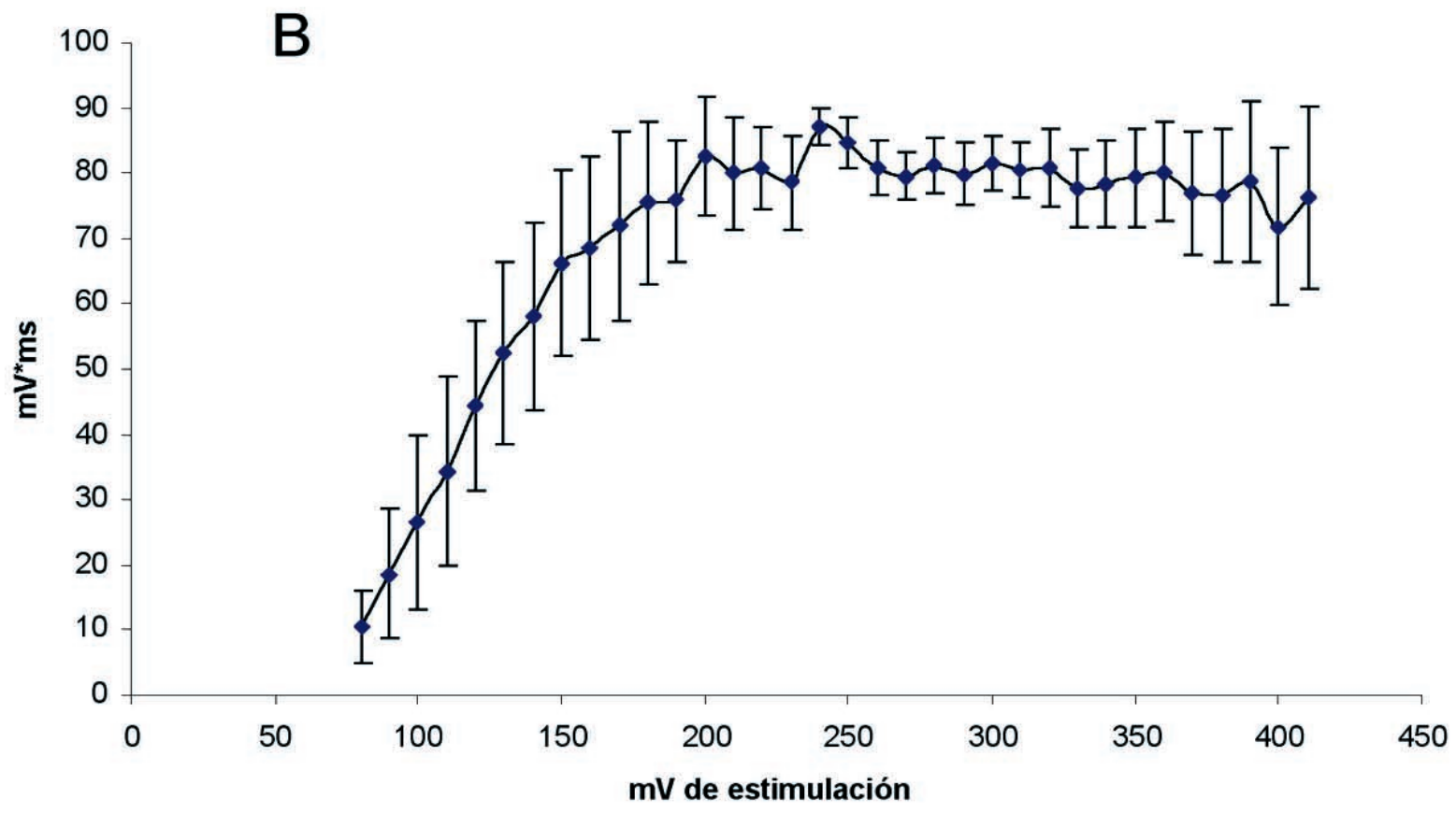

Fig. 1. Representa las características de la amplitud máxima de respuesta neuronal en Bufus marinus (A) y humanos (C) y el área bajo la curva de la respuesta Bufus marinus (B) y humanos (D) en función de diferentes intensidades de estimulación. 
Amplitud de la respuesta en función de la intensidad del estímulo

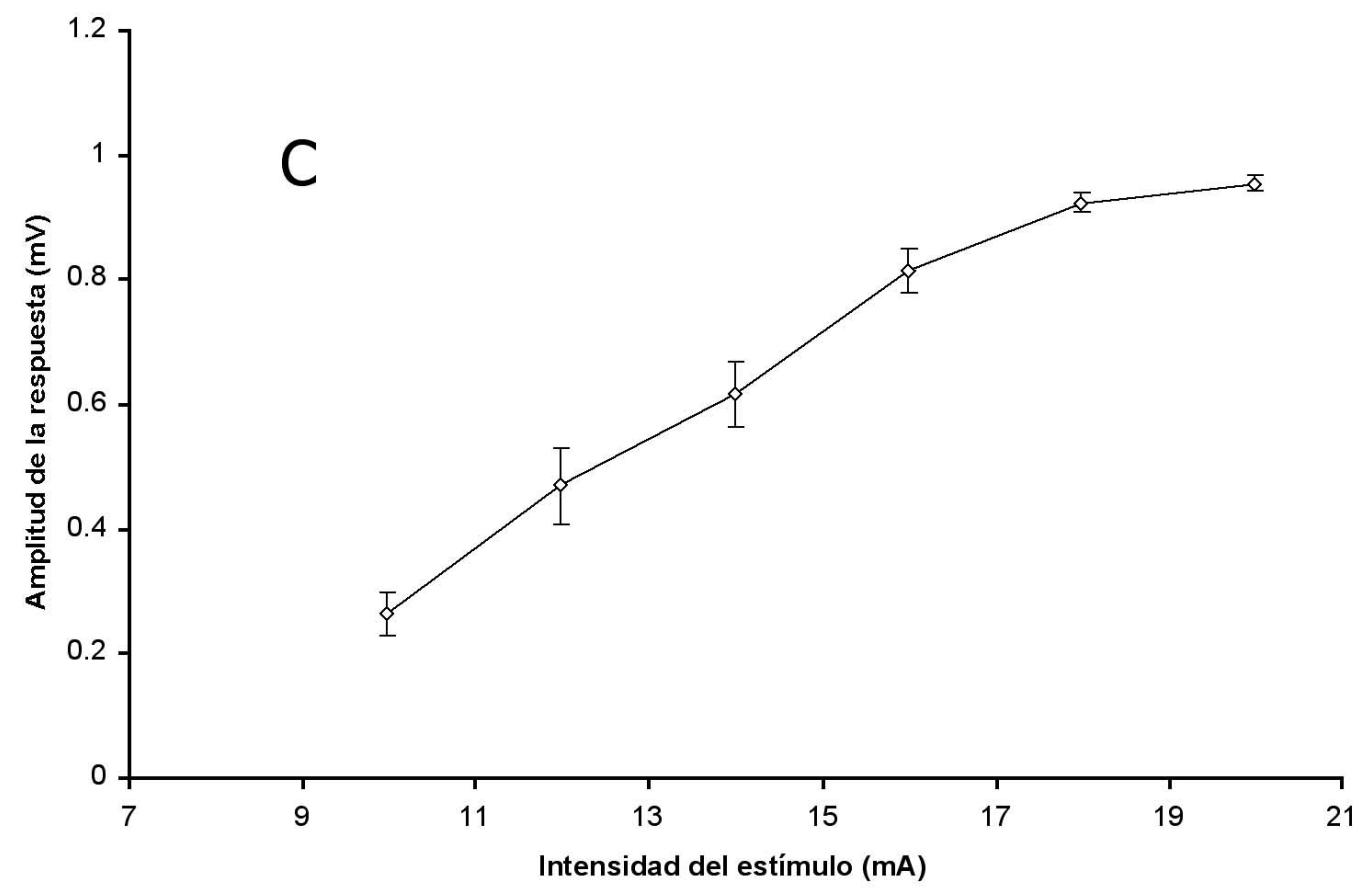

Area bajo la curva de la respuesta en función de la intensidad del estímulo

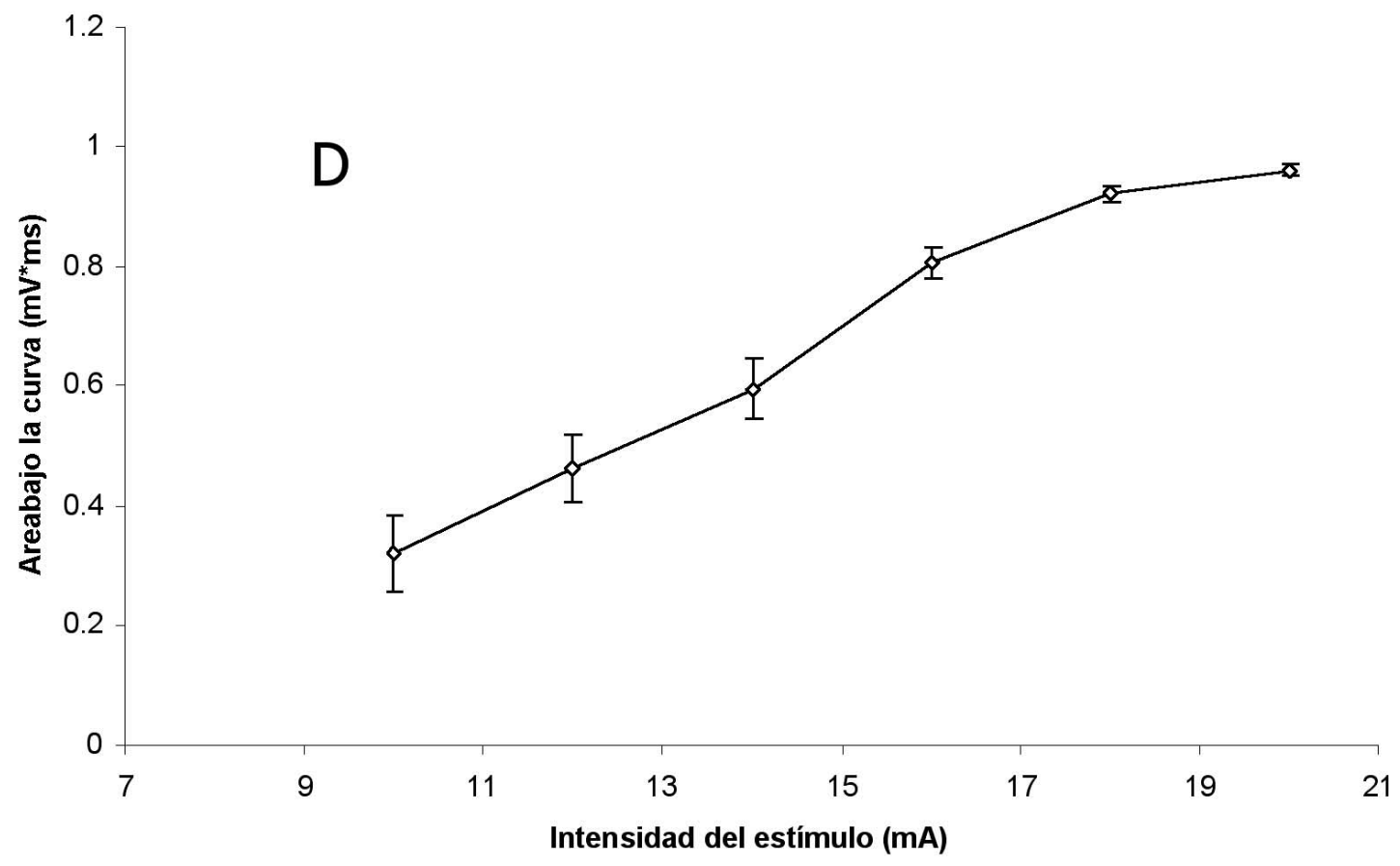


Máximas velociadades de despolarización y de repolarización

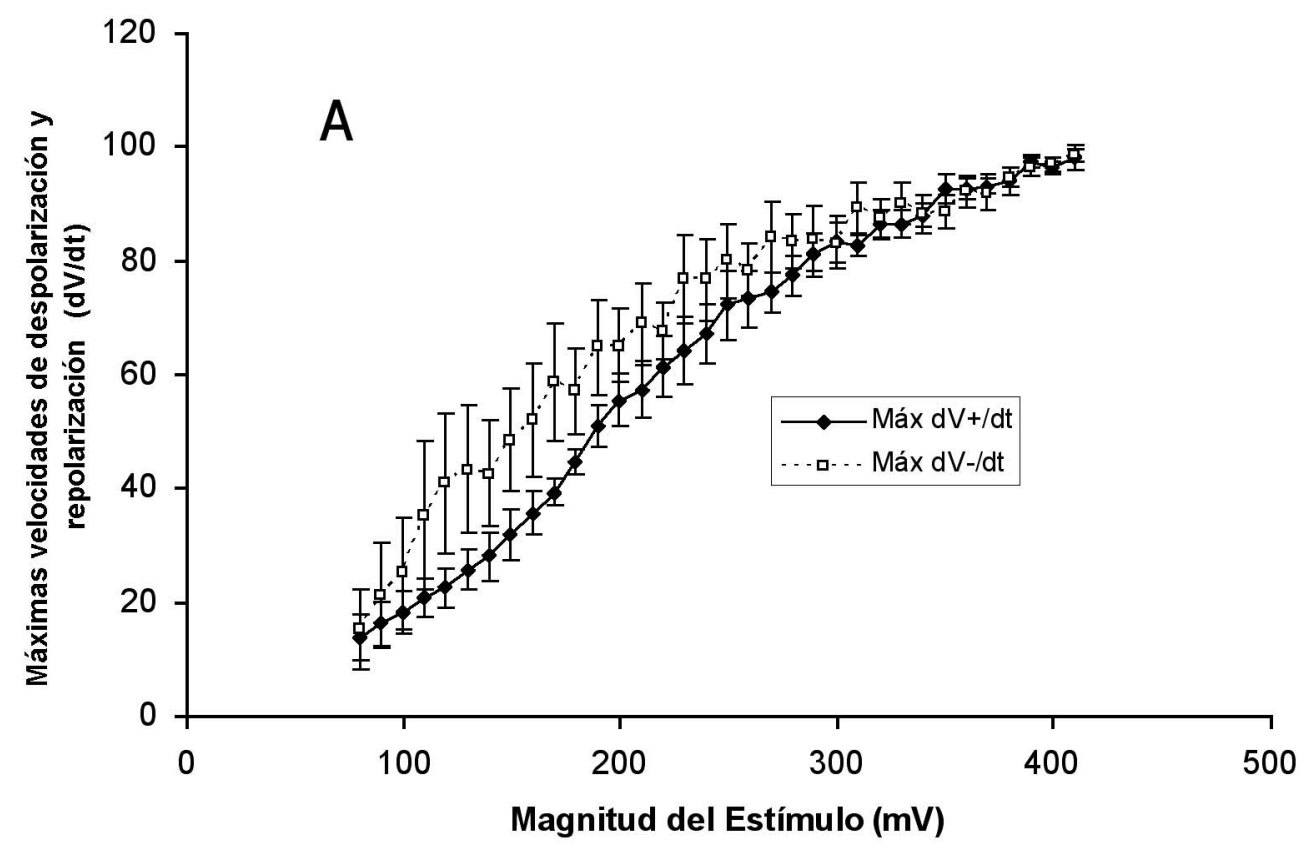

\section{Velocidades máximas de despolarización y repolarización en} función de la intensidad de estimulación

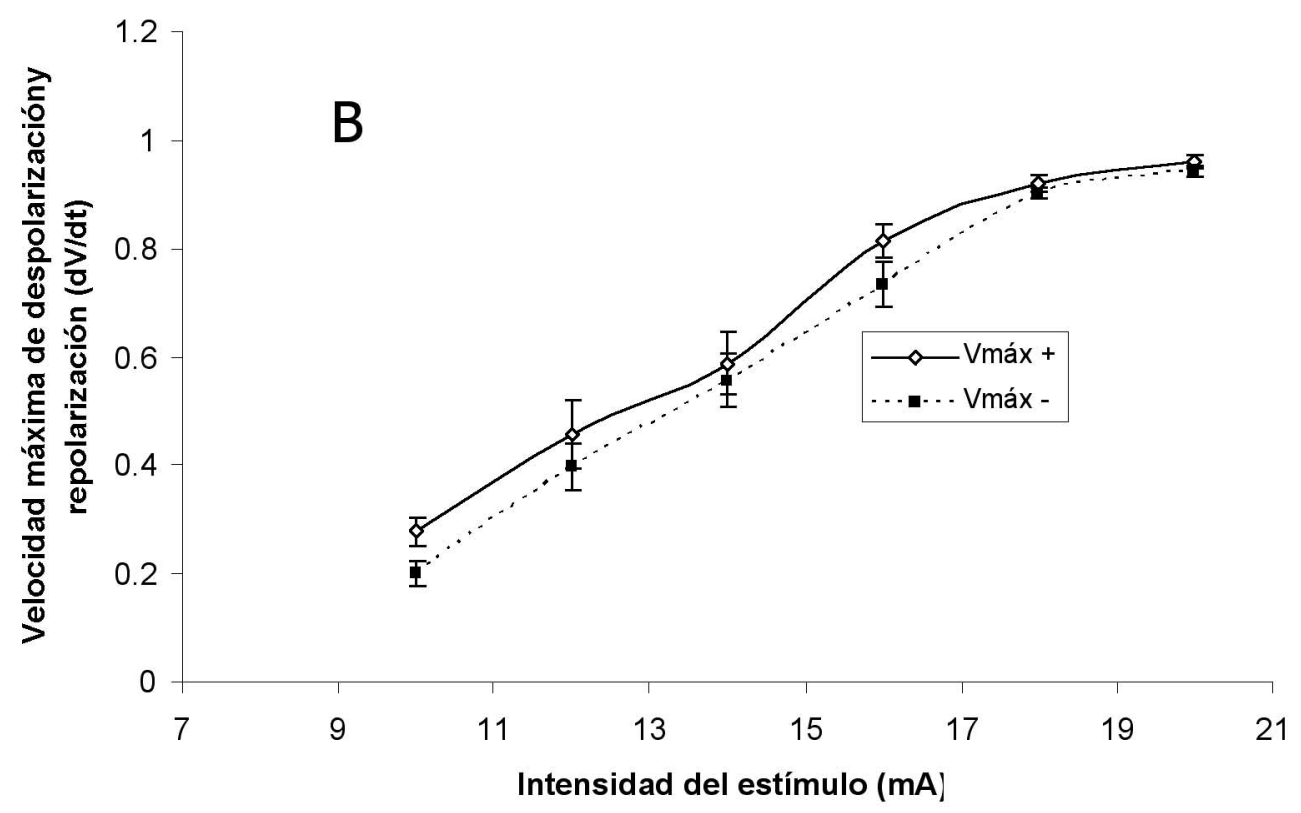

Fig. 2. Representación de las máximas velocidades de despolarización (dV+/dt) y repolarización (dV-/dt) en función de la magnitud del estímulo en Bufus marinus (A) y humanos (B). 


\section{Correlación entre velocidades máximas de despolarización J repolarización}

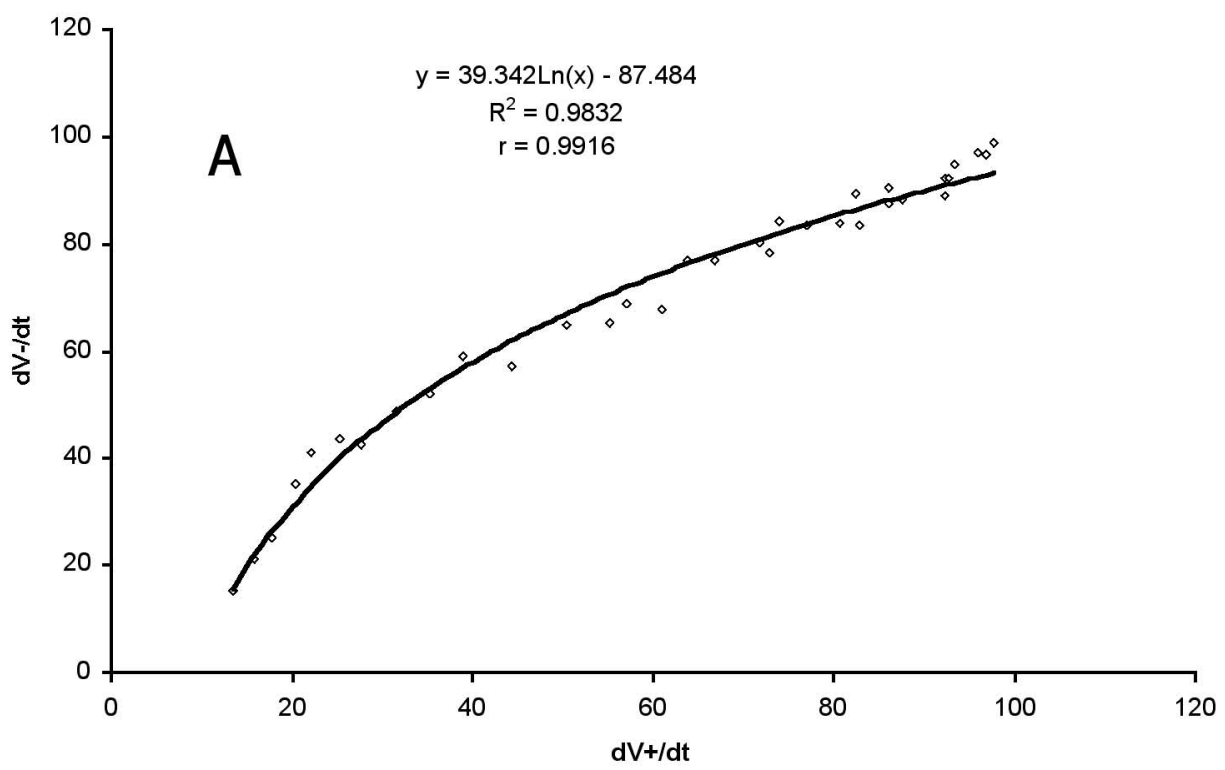

Relación entre amplitud máxima y área bajo la curva de las respuestas

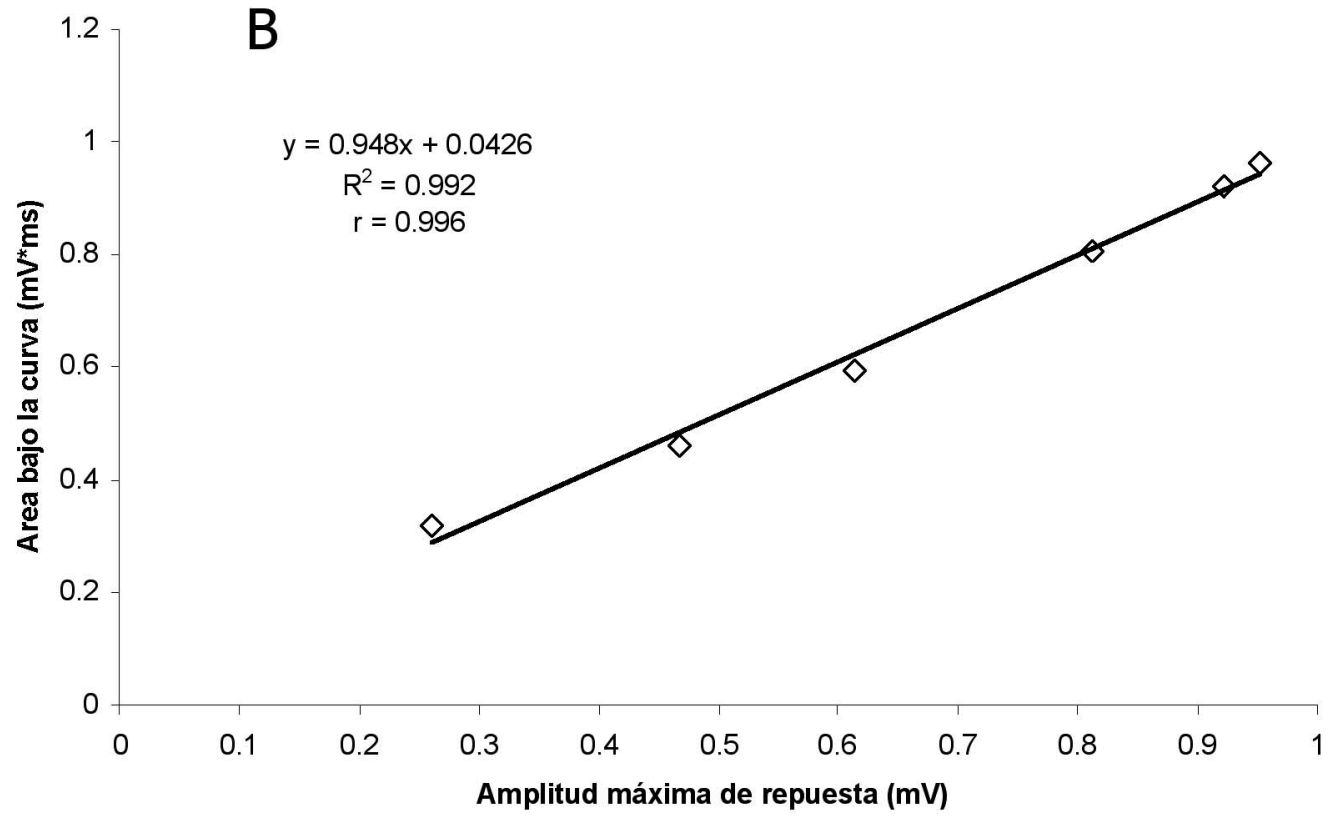

Fig. 3. Ajuste a una función logarítmica entre las máximas velocidades de despolarización (dV+/dt) y de repolarización (dV-/dt) en Bufus marinus (A) y humanos (B). 


\section{Correlación entre la máxima amplitud y el área bajo la curva de I respuesta}

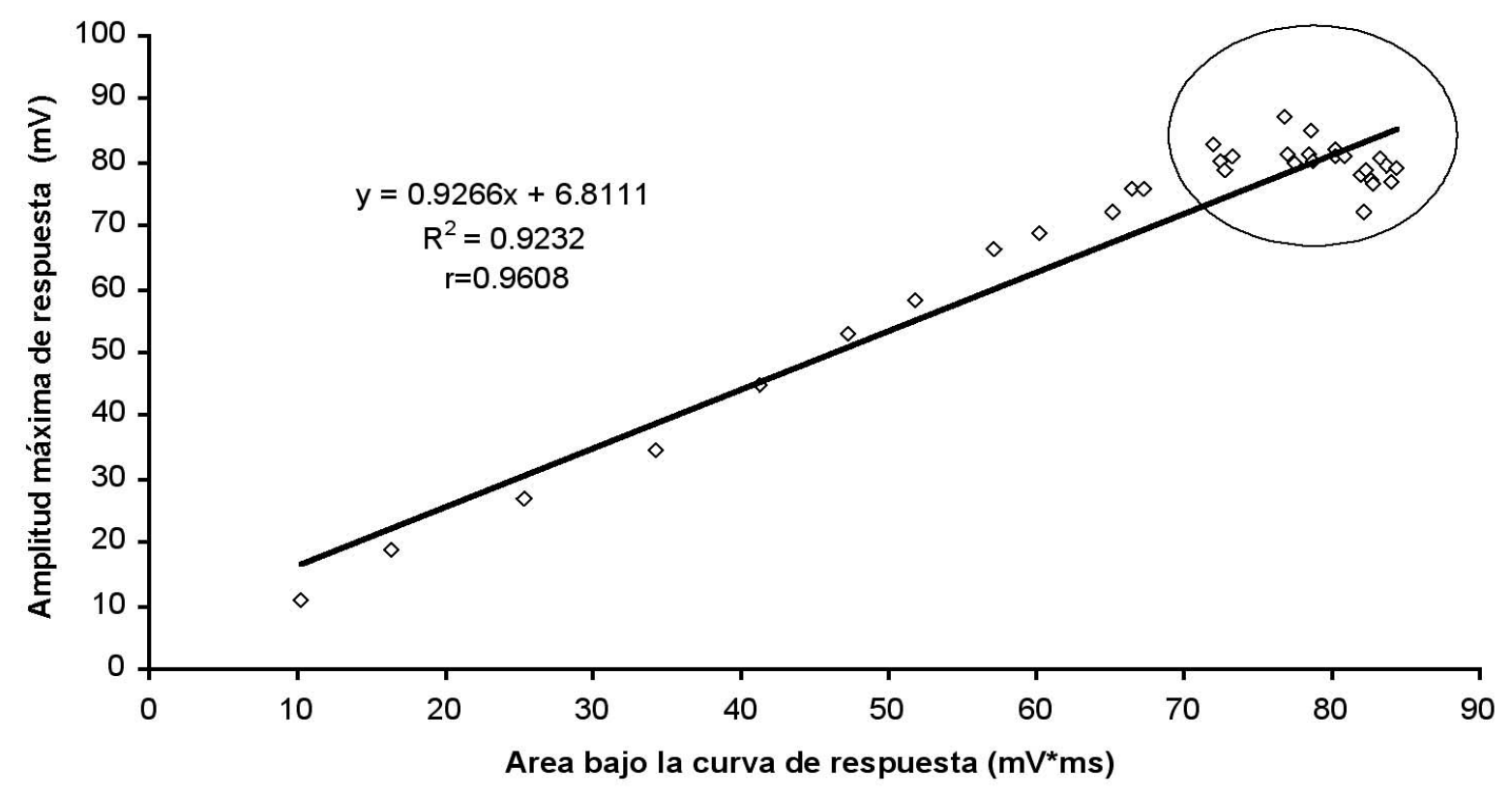

Fig. 4. Relación entre la máxima amplitud de respuesta y el área bajo la curva de la misma. Los puntos entre el círculo resalta cómo las máximas amplitudes de respuesta tienden a "saturarse" mientras que el área bajo la curva aún puede continuar incrementando.

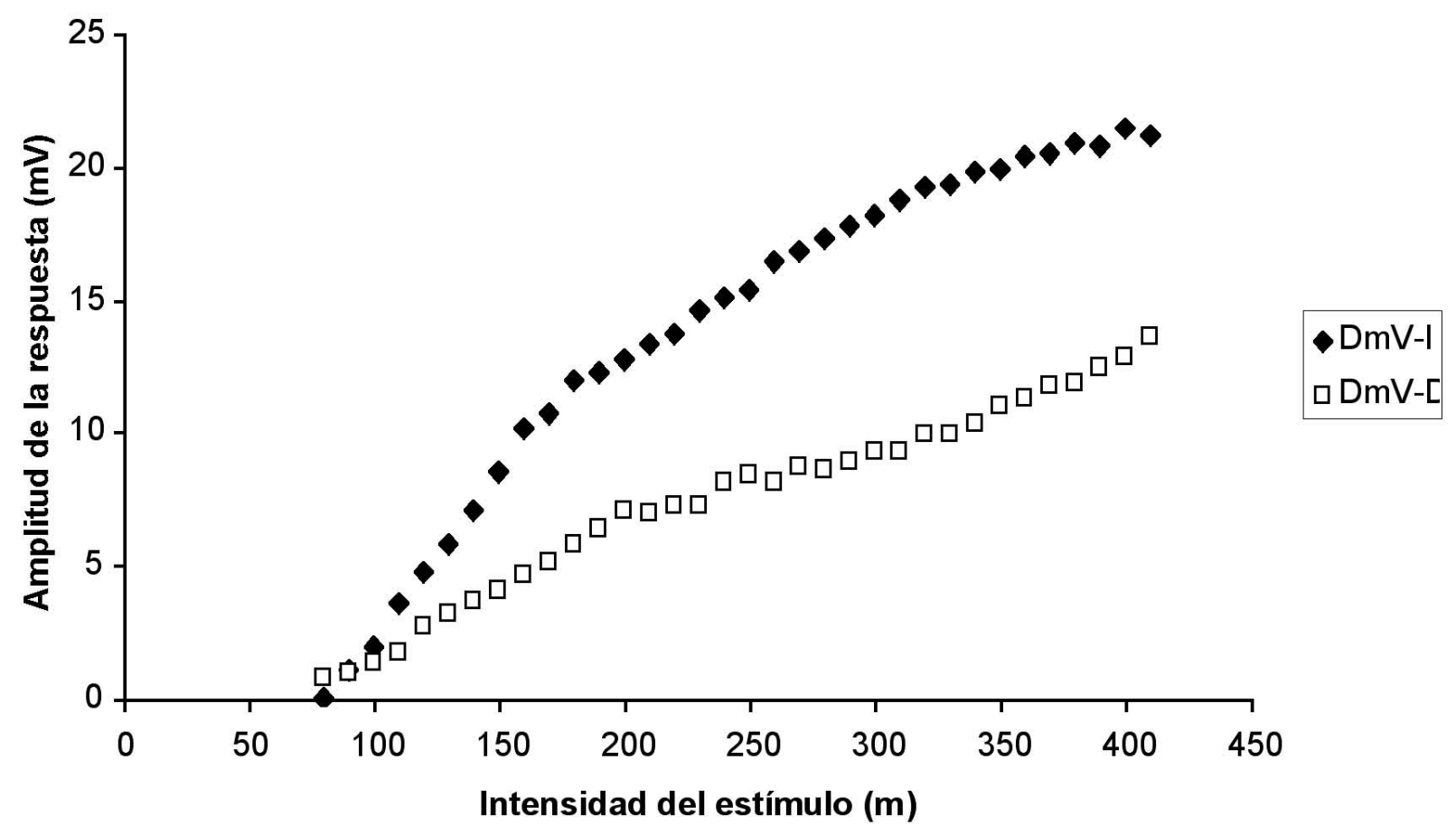

Fig. 5. Amplitud de la respuesta a diferentes estímulos en ciáticos derecho e izquierdo del mismo espécimen. 
mayor cantidad de fibras nerviosas (Fig. 1) de Bufo marinus y humanos. Las respuestas en Bufo marinus llegaron a un crecimiento estable (sin haber una correlación lineal a diferencia de humanos) que representa la saturación de las respuestas en ascenso a diferentes grados de estímulos máximos (Fig. 4). Hay que matizar que el grado de dispersión de estas respuestas fue evidente aunque sería interesante compararlas con un tamaño de muestra mayor. Las amplitudes de respuesta máxima y áreas bajo la curva son muy similares lo que pareciera representar respuestas homologas (nervios mixtos) correspondientes a velocidades de conducción de nervios rápidos tipo Alfa (Propiocepción y Motoneuronas) que parecen esconder las respuestas de nervios de menor diámetro y condición mielínica.

En relación a las velocidades máximas de despolarización $(\mathrm{dV}+/ \mathrm{dt})$ y repolarización (dV-/dt) (Fig. 1) llama la atención que en conjunto a intensidades submáximas, las velocidades máximas de repolarización del nervio aislado presentan una tendencia a ser mayores que las velocidades máximas de despolarización, lo que no ocurre con las respuestas del nervio ulnar de humanos. Por otro lado, la relación entre las velocidades máximas de despolarización y repolarización en el nervio del sapo ajusta significativamente a una tendencia logarítmica, sugiriendo que las altas velocidades máximas de despolarización pueden incrementar más que las de repolarización. Esto no ocurre con las respuestas del nervio ulnar, donde esta relación ajustó mejor a una tendencia lineal. Este fenómeno aunque interesante en conjunto no parece tener alcances significativos individualmente debido a la dispersión de los datos y lo expresa la correlación de estas velocidades máximas de despolarización y repolarización cuando son representadas bajo una función logarítmica y lineal en ciático y nervio lunar respectivamente (sapos R2 $=0.9832$, humanos $\mathrm{R} 2=0.0 .992$ ) (Fig. 3). Estos resultados son apoyados por las correlaciones a dos colas $(\mathrm{p}=0.01)$ entre las variables evaluadas del nervio ciático y ulnar (Tabla I). Es posible que a intensidades submáximas, las velocidades máximas de repolarización del nervio aislado se comporten diferentes a las de despolarización debido a mecanismos de permeabilidad de membranas en los que los canales iónicos podrían tener velocidades de apertura y cierre en la despolarización. (Fry, 2005; Kobayashi et al., 1998).

Llama particularmente la atención cómo las amplitudes de respuesta a potenciales evocados varían entre ciáticos del mismo espécimen (Fig. 5). Una explicación probable es brindada por Sandén et al. (2005) e igualmente por Wei et al. quienes muestran como la extremidad dominante tiene un rendimiento nervioso (velocidades de conducción y amplitud de respuesta) superiores. (Sandén et al.; Havton et al., 2007)

\begin{tabular}{lllll}
\hline & Amplitud Máx. & Área & Máx. dV+/dt & Máx. dV-/dt \\
\hline Amplitud Máx. & 1000 & & & \\
Área & 0,961 & 1000 & & \\
Máx. dV+/dt & 0,908 & 0,773 & 1000 & 1000 \\
Máx.dV-/dt & 0,962 & 0,858 & 0,981 & \\
& +-0.436 & Valor Crítico 0,01 & dos colas & \\
& 34 & Tamaño Muestra & & \\
& &
\end{tabular}

\begin{tabular}{lllll}
\hline & Amplitud Máx. & Área & V Máx. + & V Máx. - \\
\hline Amplitud Máx. & 1000 & & \\
Área & 0,996 & 1000 & \\
V Máx.+ & 0,998 & 0,999 & 1000 & \\
V Máx.- & 0,997 & 0,997 & 0,997 & 1000 \\
& +-0.917 & Valor Crítico 0,01 & dos colas & \\
& 6 & Tamaño Muestra & \\
\hline
\end{tabular}


La velocidad de conducción, la amplitud de respuesta, la dispersión temporal y las latencias entre otras características nerviosas son un tópico polémico desde un punto de vista de utilidad clínica. La ventaja de esta información se encuentra en la posibilidad de caracterizar polineuropatías axonales y desmielinizantes (causadas por micro o macrotrauma de atrapamiento, diabetes, Guillan Barre, esclerosis, lepra, síndrome de Raynaud, isquemia, etc.) y que generan disfunciones nerviosas que pueden ser diagnosticadas y tratadas a tiempo (Caliandro et al.; Hvton et al.; Kiziltan et al., 2007; Severinsen \& Andersen, 2007; Tankisi et al., 2007; Wei et al., 2005; Husain \& Malaviya, 2007; Ballesteros \& Ramirez, 2007; Lew et al., 2005; Chang et al., 2004; Colak et al., 2005; Kostopoulos et al., 2008; Mc Dermontt et al., 2006; Chang et al., 2006a; 2006b). La disminución de la velocidad de conducción y de la amplitud de respuesta sumada a un aumento de las latencias de respuesta y de su dispersión temporal es involucrada en este tipo de patologías. Por lo general factores funcionales, morfológicos, metabólicos y asociados a estas enfermedades (la disminución de ATPasa en la bomba $\mathrm{Na}+/ \mathrm{K}+$, aumento de la glicólisis anaeróbica, el incremento de la glicolisacion no enzimática proteínica, la vía del sorbitol-aldosa reductasa, la disminución del contenido de mioinositol, la disminución del soporte nuerotrófico, la hiperosmolaridad, el daño vascular por proteína kinasa $\mathrm{C}$, los radicales libres por auto-oxidación de la glucosa, la isquemia e hipoxia neural debido a microangiopatia, la atrofia axonal retrograda, entre otros.), terminan por producir cambios en el rendimiento nervioso del organismo (Kikkawa et al., 2005; Saini et al., 2004; Padua et al., 2007).
Finalmente, consideramos que el equipo que se usó para registrar estas respuestas y características del potenciales de acción compuesto de fibras nerviosas mixtas (sensoriales y motoras) utilizó electrodos de superficie que miden eficientemente las características de los potenciales de acción compuesto pero no permiten una identificación optima de las propiedades de conducción en general en casos de neuropatías que no involucran todas las fibras del nervio. En este sentido, principalmente se registraron las respuestas de conducción nerviosa más rápidas a cargo de la población neuronal motora alfa (Sanden et al.).

\section{CONCLUSIÓN}

Detectar neuropatías en estadios tempranos de daño mielínico es vital para un tratamiento oportuno y debido a que el daño se manifiesta cuando el conjunto de neuronas se hacen disfuncionales. El uso de pruebas electrofisiológicas como herramienta de diagnostico muestra ser de gran utilidad en la identificación de factores predisponentes e iniciadores de algunas patologías asociadas al deterioro neuronal musculo-esquelético.

\section{AGRADECIMIENTOS}

Expreso mi reconocimiento al Dr. Carlos Conde Cotes por su valiosa asistencia en la experimentación y edición de este artículo.

RAMÍREZ, L M. Evaluation of the effects of various intensities of nerve stimulation on the scale of the response in different species. Int. J. Morphol., 28(1):227-238, 2010.

SUMMARY: Nervous behaviour by electrical stimulation has been widely studied since 1840, times that initiated the comprehension of the conduction velocity and nerve's amplitude responses in animals. The amplitude response in nerve segments electrically stimulated is generated by the result of composed action potentials. This research pretended to study the nerve responses to evoked potentials in human in vivo (ulnar nerve) and in vitro in animals (Bufus marinus) in sciatic nerve. Electrical stimulation resulted in rising responses of heterogeneous quality inter and intra-species. Mechanisms involved in each response are examined.

KEY WORDS: Composed action potential; Stimulation electrode; Register electrode; stimulation impulse; Intensity of charge displaced.

\section{REFERENCIAS BIBILOGRÁFICAS}

Bean, B. P. The action potential in mammalian central neurons. Nat. Rev. Neurosci., 8:451-65, 2007.

Ballesteros, L. E. \& Ramirez, L. M. Possible implications of an accessory abductor digiti minimi muscle: a case report. J. Brachial Plex. Peripher. Nerve Inj., 2:22, 2007.
Caliandro, P.; Stålberg, E.; La Torre, G. \& Padua, L. Sensitivity of conventional motor nerve conduction examination in detecting patchy demyelination: a simulated model. Clin. Neurophysiol., 118:1577-85, 2007. 
Chang, M. H.; Liu, L. H.; Lee, Y. C.; Wei, S. J.; Chiang, H. L. \& Hsieh, P. F. Comparison of sensitivity of transcarpal median motor conduction velocity and conventional conduction techniques in electrodiagnosis of carpal tunnel syndrome. Clin, Neurophysiol., 117:984-91, 2006.

Chang, M. H.; Liu, L. H.; Wei, S. J.; Chiang, H. L. \& Hsieh, P. F. Does retrograde axonal atrophy really occur in carpal tunnel syndrome patients with normal forearm conduction velocity? Clin. Neurophysiol., 115:27838, 2004.

Chang, C. W.; Shieh, S. F.; Li, C. M.; Wu, W. T. \& Chang, K. F. Measurement of motor nerve conduction velocity of the sciatic nerve in patients with piriformis syndrome: a magnetic stimulation study. Arch Phys Med Rehabil., 87:1371-5, 2006.

Colak, T.; Bamaç, B.; Gönener, A.; Ozbek, A. \& Budak, F. Comparison of nerve conduction velocities of lower extremities between runners and controls. J. Sci. Med. Sport, 8:403-10, 2005.

Frank, R. G. Jr. The Columbian exchange: American physiologists and neuroscience techniques. Fed. Proc., 45:2665-72, 1986.

Fry, C. H. Action potential and nervous conduction. Surgery (Oxford), 23:425-429, 2005.

Fukuda, H.; Sonoo, M.; Kako, M. \& Shimizu, T. Optimal method to determine the stimulus intensity for median nerve somatosensory evoked potentials. J. Clin. Neurophysiol., 24:358-62, 2007.

Guyton, A. C. Tratado de Fisiología Médica. 11ª Ed. Madrid, Elsevier, 2006

Havton, L. A.; Hotson, J. R. \& Kellerth, J. O. Correlation of median forearm conduction velocity with carpal tunnel syndrome severity. Clin. Neurophysiol., 118:781-5, 2007.

Husain, S. \& Malaviya, G. N. Early nerve damage in leprosy: an electrophysiological study of ulnar and median nerves in patients with and without clinical neural deficits. Neurol. India, 55:22-6, 2007.

Kikkawa, Y.; Kuwabara, S.; Misawa, S.; Tamura, N.; Kitano, Y.; Ogawara, K. \& Hattori, T. The acute effects of glycemic control on nerve conduction in human diabetics. Clin. Neurophysiol., 116:270-4, 2005.
Kiziltan, M. E.; Gunduz, A.; Kiziltan, G.; Akalin, M. A. \& Uzun, N. Peripheral neuropathy in patients with diabetic foot ulcers: clinical and nerve conduction study. J. Neurol. Sci., 258:75-9, 2007.

Kobayashi, J.; Ohta, M. \& Terada, Y. Differential contribution of $\mathrm{Na}+\mathrm{K}+$ pump and $\mathrm{K}+$ conductance to the post-tetanic hyperpolarization of the subtypes of tetrodotoxin-resistant C-fibers in the isolated bullfrog sciatic nerve. Neurosci. Lett., 242:57-60, 1998.

Kostopoulos, D. C.; Rizopoulos, K. \& Vartholomeos, N. Nerve conduction velocities in the lower extremity in patients with Raynaud's phenomenon and clinical applications. J. Bodyw. Mov. Ther., 12:58-66, 2008.

Lew, H. L.; Date, E. S.; Pan, S. S.; Wu, P.; Ware, P. F. \& Kingery, W. S. Sensitivity, specificity, and variability of nerve conduction velocity measurements in carpal tunnel syndrome. Arch. Phys. Med. Rehabil., 86:12-6, 2005.

Nakanishi, T.; Tamaki, M.; Mizuwawa, H.; Akatsuka, T. \& Kinoshita, T. An experimental study for analyzing nerve conduction velocity. Electroencephalogr. Clin. Neurophysiol., 63:484-7, 1986.

McDermott, M. M.; Sufit, R.; Nishida, T.; Guralnik, J. M.; Ferrucci, L.; Tian, L.; Liu, K.; Tan, J.; Pearce, W. H., Schneider, J. R.; Sharma, L. \& Criqui, M. H. Lower extremity nerve function in patients with lower extremity ischemia. Arch. Intern. Med., 166:1986-92, 2006.

Morélot-Panzini, C.; Fournier, E.; Donzel-Raynaud, C.; Dubourg, O.; Willer, J. C. \& Similowski, T. Conduction velocity of the human phrenic nerve in the neck. $J$. Electromyogr. Kinesiol., 19(1):122-30, 2009.

Padua, L.; Caliandro, P. \& Stålberg, E. A novel approach to the measurement of motor conduction velocity using a single fibre EMG electrode. Clin. Neurophysiol., 118:1985-90, 2007.

Saini, A. K.; Arun, K. H.; Kaul, C. L. \& Sharma, S. S. Acute hyperglycemia attenuates nerve conduction velocity and nerve blood flow in male Sprague-Dawley rats: reversal by adenosine. Pharmacol. Res., 50:5939,2004

Sandén, H.; Edblom, M.; Hagberg, M. \& Wallin, B. G. Bicycle ergometer test to obtain adequate skin temperature when measuring nerve conduction velocity. Clin. Neurophysiol., 116:25-7, 2005. 
RAMíREZ, L M. Evaluación de los efectos de diversas intensidades de estimulación nerviosa sobre la amplitud de la respuesta en diferentes especies. Int. J. Morphol., 28(1):227-238, 2010.

Severinsen, K. \& Andersen, H. Evaluation of atrophy of foot muscles in diabetic neuropathy -- a comparative study of nerve conduction studies and ultrasonography. Clin. Neurophysiol., 118:2172-5, 2007.

Schuhfried, O.; Angst, M.; Herceg, M. \& Paternostro-Sluga, T. Interexaminer repeatability of antidromic ulnar sensory nerve conduction velocity measurements. Arch. Phys. Med. Rehabil., 86:2047-50, 2005.

Tankisi, H.; Pugdahl, K.; Johnsen, B. \& FuglsangFrederiksen, A. Correlations of nerve conduction measures in axonal and demyelinating polyneuropathies. Clin. Neurophysiol., 118:2383-92, 2007.

Wei, S. H.; Jong, Y. J. \& Chang, Y. J. Ulnar nerve conduction velocity in injured baseball pitchers. Arch. Phys. Med. Rehabil., 86:21-5, 2005.
Dirección para correspondencia:

Dr. Luis Miguel Ramírez Aristeguieta

Departamento de Ciencias Básicas,

Facultad de Odontología

Universidad Santo Tomas de Aquino

Bucaramanga

COLOMBIA

Email: Imra3@yahoo.com

Recibido : 08-09-2009

Aceptado: 09-02-2010 